\title{
Thermodynamic analysis of processes creating the defects in cadmium telluride crystals under conditions of high-temperature annealing
}

\author{
V.V. Prokopiv ${ }^{1}$, P.M. Fochuk ${ }^{2}$, I.V. Gorichok ${ }^{1}$, E.V. Vergak ${ }^{2}$ \\ ${ }^{l}$ V. Stefanyk Precarpathion National University, 57, Shevchenko str., \\ 76000 Ivano-Frankivsk, Ukraine; e-mail: goritchok@rambler.ru \\ ${ }^{2}$ Institute of Inorganic Chemistry, Chernivtsi National University, 2, Kotsiubynsky str., \\ 74012 Chernivtsi, Ukraine; e-mail:fochukp@gmail.com
}

\begin{abstract}
The defective structure of specifically undoped cadmium telluride crystals was researched using the theory of thermodynamic potentials. The calculated concentration of point defects and free charge carriers in the CdTe crystals, depending on technological factors of two-temperature annealing (annealing temperature $T$ and partial pressure of cadmium $P_{\mathrm{Cd}}$ vapor). The dominant types of defects that determine the basic properties of the material $n$ - and $p$-type conduction were determined.
\end{abstract}

Keywords: two-temperature annealing, thermodynamic potential, point defects, electrical properties, cadmium telluride.

Manuscript received 07.08.09; accepted for publication 10.09.09; published online 30.10.09.

\section{Introduction}

As the point defects determine basic properties of semiconductor materials, their characteristics can be studied by modeling their imperfect structure.

To calculate the equilibrium concentration of defects, the method of quasi-chemical reactions by Kreger is mainly used [1-8], empirically determined equilibrium constants $K_{0}$ and enthalpies for reactions of defects formation $\Delta H$ are being used in it. But considerable interest is caused by the works [9-11] where researches of point defects in the structure were performed using the theoretically determined energies of defects formation and change in the vibration frequency of atoms in the vicinity of the defect at its creation. The use of thermodynamic parameters of defects defined $a b$ initio allows to calculate constants of equilibrium for the subsequent comparison with various experimental results. The latter often have considerable variations, especially in the cases when concentrations of a few dominant defects are close to each other. Also, this calculation is especially important for situations where constants are not determined or to do this in experiments is quite difficult.

Without regard, that in the works [9-11], with the use of theoretically determined parameters of defects [12], satisfactory accordance of the calculated and experimentally measured concentrations of charge carriers was obtained. But in our opinion, the parameters of some defects (energies of creation and energy of ionization) are not fully corresponding to experimental data, as it follows from the analysis presented in the work.

Thus, the problem to determine the influence of lattice disordering on properties of cadmium telluride crystals remains topical. The purpose of this work is to study the influence of two-temperature annealing technological condition on creation of the defect structure in crystals and their electric properties. To numerically calculate the equilibrium concentrations of point defects under conditions of high temperature annealing in vapors of components, we did not use any quasi-chemical formalism but instead the general thermodynamic equation of phase equilibrium in the heterogenic system.

\section{Method of experiment}

The crystals for investigations were grown by the Bridgman method. For a synthesis, we used the cadmium КД-0000 and tellurium - ТВ-4 (according to a certificate, content of basic substance was not less than $99.9999 \%$ and $99.9997 \%$, accordingly), additionally cleaned by the method of zone melting. The concentration of non-controlled background impurities, as a rule, did not exceed $10^{-5}-10^{-6}$ wt. $\%$. 
The high temperature measurements of the Hall effect were performed using the samples placed into a vacuum ampoule in a two-area stove, where the first area provided the temperature of samples, and the second one - temperature of $\mathrm{Cd}$. Adjustment of both areas was carried out by a computer that received a signal from two thermocouples located, accordingly, near the sample and more cold end of ampoule, the temperature of which determined the pressure of $\mathrm{Cd}$ vapors. The accuracy of keeping the temperature was $\pm 0.5 \mathrm{~K}$. The temperature of both areas could be linearly changed within the wide interval of speeds, both when heating and cooling. Managing the whole commutation and taking the signals from all pairs of contacts and thermocouples was carried out by a computer and voltmeter.

\section{Method to determine the equilibrium concentration of defects}

The equilibrium concentrations of point defects in a crystal under two-temperature annealing are determined from the condition of equality of chemical potentials for every component in all phases of the system:

$\mu_{i}^{s}=\mu_{i}^{g}$, or $d G_{i}^{s} / d N_{i}^{s}=d G_{i}^{g} / d N_{i}^{g}$,

where $G^{s}, G^{g}$ and $N^{s}, N^{g}$ are Gibbs thermodynamic potentials and concentrations of particles in a crystal and gas, accordingly; $i-\mathrm{Cd}$, Te.

The Gibbs energy of crystal:

$$
G=\sum\left(E+E_{v i b}\right)[D]+n E_{c}-p E_{v}-T\left(S_{n}+S_{p}+S_{k}\right)
$$

where $E$ is the energy of defect creation; $F_{v i b}$ is the free defect vibration energy; $[D]$ is the concentration of the defect $D ; n$ and $p$ are concentrations of electrons and holes; $E_{c}, E_{v}$ are energies of the conductivity band bottom of and top of the valence band, $S_{k}$ is the configuration entropy, $S_{n}$ and $S_{p}$ are entropies of electrons in the conductivity band and holes in the valence band.

Summation was performed over all sublattices and all defects in each sublattice. Energies of singly and doubly ionized defects were determined using the formulas:

$$
E_{1}=E_{0}-\frac{Z}{|Z|} \varepsilon_{1}, \quad E_{2}=E_{0}-\frac{Z}{|Z|}\left(\varepsilon_{1}+\varepsilon_{2}\right),
$$

where $E_{0}$ is the energy of creation of a neutral defect; $Z$ is the charge state of the defect; $\varepsilon_{1}, \varepsilon_{2}$ are the first and second levels of defect ionization.

A change of the free vibration energy at defect creation was determined using the formula [13]:

$$
F_{v i b}= \pm\left(3 k T \ln \left(T_{\theta} / T\right)-k T\right)+x \cdot 3 k T \ln \left(\omega / \omega_{0}\right) \text {. }
$$

$T_{\theta}$ is the Debay temperature, $x$ is an amount of atoms changing the frequency of intrinsic vibrations from $\omega_{0}$ to $\omega$.
The entropy was determined by the Boltzmann law:

$S_{k}=k \ln \left(\Pi W_{j}\right)=\sum k \ln \left(W_{j}\right)=\sum S_{j}$,

where $W_{j}$ is the thermodynamic probability of $j$ sublattice. For a sublattice with several different types of defects:

$W_{j}=\frac{J !}{(J-\Sigma[D]) ! \cdot \prod[D] !}$,

where $J$ is the concentration of knots where defects can appear.

For electrons and holes, thermodynamic probabilities are evened:

$W_{n}=\frac{N_{c} !}{\left(N_{c}-n\right) ! n !}, W_{p}=\frac{N_{v} !}{\left(N_{v}-p\right) ! p !}$,

where

$n=N_{c} \exp (\mu / k T), p=N_{v} \exp \left(-\left(\mu+E_{g}\right) / k T\right)$,

$N_{c}, N_{v}$ is the density of states in the conductivity band and in the valence band, accordingly, $E_{g}$ is the bandgap energy, $\mu$ is the chemical potential of electrons determined from the equation of electro-neutrality:

$N_{c} \exp (\mu / k T)-N_{v} \exp \left(-\left(\mu+E_{g}\right) / k T\right)=\sum Z \cdot\left\lfloor D^{z}\right\rfloor$.

The chemical potential of defect, which equals to the chemical potential of component taken with the sign "+" or "-" depending on the type of defects, was determined by differentiation of the Gibbs energy with regard to the concentration of defects.

$$
\begin{aligned}
& \mu_{D_{i}}^{s}=E_{D_{i}}-k T \cdot \ln \left(\frac{J-\sum[D]}{\left[D_{i}\right]}\right)+ \\
& +\left[n\left(\frac{E_{c}}{k T}-\ln \left(\frac{N_{c}-n}{n}\right)\right)+p\left(\frac{E_{v}}{k T}-\ln \left(\frac{N_{v}-p}{p}\right)\right)\right] \times \\
& \frac{k T \cdot Z_{D_{i}}}{\sqrt{\left(\sum Z[D]\right)^{2}+4 N_{c} N_{v} \exp \left(-E_{g} / k T\right)}} .
\end{aligned}
$$

The chemical potential of gas [14]:

$$
\mu^{g}=k T \ln P+\mu_{0} \text {. }
$$

In the case of monoatomic gas $\mathrm{Cd}$ :

$\mu_{0}=k T\left[-\ln (k T)+\ln \left(h^{3} /(2 \pi m k T)^{3 / 2}\right)\right]$.

For diatomic gas $\mathrm{Te}_{2}$ :

$\mu_{0}=k T\left\lfloor-\ln (k T)+\ln \left(h^{3} /(2 \pi m k T)^{3 / 2}\right)+\right.$

$\left.+\ln \left(h^{2} / 8 \pi^{2} I k T\right)+\ln (h v / k T)\right]$.

Here, $m$ is the mass of atom or molecule, $I=m l^{2}$ is the moment of molecule inertia, $l$ is the inter-kernel distance in the molecule, $v$ is the internal frequency of molecular vibrations. 
Thus, we got the system of equations of the type $\pm \mu_{D_{i}}^{s}=\mu_{i}^{g} \quad$ allowing to find the equilibrium concentration of defects, which was solved by minimization of the quadratic function from deficiencies $\left( \pm \mu_{D_{i}}^{s}-\mu_{i}^{g}\right)[15]$ :

$$
L_{\min }=\sum\left( \pm \mu_{D_{i}}^{s}-\mu_{i}^{g}\right)^{2} \text {. }
$$

The co-ordinates of a function minimum $L_{\min }$ are the equilibrium values of defect concentrations at the set pressure $P$ and temperature $T$. To find concentrations of electrons and holes, we used formulas (8) where the chemical potential of electrons was determined using the formula (10).

Vacancies and intersticial atoms of $\mathrm{Cd}$ and Te such as $V_{\mathrm{Cd}}, V_{\mathrm{Te}}, \mathrm{Cd}_{i}, \mathrm{Te}_{i}$ were considered as the main atomic defects. Each of these defects can be in three charge states: neutral, singly or doubly charged. Thus, $L_{\min }$ is a function of twelve variables.

The parameters of point defects that are used in calculations are shown in Table.

\section{Energies of defects creation and energy of their ionization}

Energies of defects creation are expected $a b$ initio in the works $[12,16]$. The main difference in calculations between these works consists in the choice of bases functions. In [12] we used a base built on the atomic functions of waves with $l \leq 5$, and in [16] calculation was performed on the base of flat waves. The use of different bases was stipulated by the fact that the obtained energies of defects creation considerably differ between themselves. To calculate them, we used values determined in the work [16] (Table), because majority of them differ a little from energies of creation, which we obtained in experimental [6] and calculation works [9-10].

Table. Parameters of point defects in CdTe crystals.

\begin{tabular}{|c|c|c|c|c|}
\hline & $V_{\mathrm{Cd}}$ & $\mathrm{Cd}_{i}$ & $V_{\mathrm{Te}}$ & $\mathrm{Te}_{i}$ \\
\hline$E, \mathrm{eV}$ & $2.67[16]$ & $2.04[16]$ & $3.24[16]$ & $3.41[16]$ \\
\hline \multirow{2}{*}{$\varepsilon_{1}, \mathrm{eV}$} & $E_{v}+0.05$ & $E_{c}-0.016$ & $E_{c}-0.01$ & $E_{v}+0.15$ \\
& {$[2]$} & {$[17]$} & {$[19]$} & {$[2]$} \\
\hline \multirow{2}{*}{$\varepsilon_{2}, \mathrm{eV}$} & $E_{v}+0.8$ & $E_{c}-0.17$ & $E_{c}-0.34$ & $E_{v}+0.57$ \\
& {$[12]$} & {$[18]$} & {$[18]$} & {$[16]$} \\
\hline$x$ & 4 & 5 & 4 & 5 \\
\hline$\omega / \omega_{0}$ & 3.75 & 0.25 & 1.60 & 0.15 \\
\hline
\end{tabular}

Difficult and ambiguous is the task to determine a position of energy levels of defects in the bandgap of CdTe. Information of different authors strongly differs between itself [1, 2, 12, 16-27].

For the vacancy of cadmium, from data of different authors the first level of ionization makes $E_{v}+(\approx 0.06) \mathrm{eV}$ $[2,21,23,24]$ or $E_{v}+(\approx 0.15-0.20) \mathrm{eV}[1,12,16]$. The experimentally observed level $E_{v}+(\approx 0.06) \mathrm{eV}$ is one of the most intensive, and authors related it to the first charge state of the cation vacancy. The level of
$E_{v}+(\approx 0.15) \mathrm{eV}$ is also observed experimentally [20], but not in all samples and with a less intensity. Calculations give for the first charge state of the cation vacancy the values $0.2 \mathrm{eV}$ [12] and $0.15 \mathrm{eV}$ [16]. In the work [2], the level $E_{v}+0.15 \mathrm{eV}$ is added to the first charge state of intersticial tellurium atom. Besides, the energy of ionization close to $0.15 \mathrm{eV}$ can belong to some complexes and impurity atoms [16]. The second charge state of cadmium vacancy lies within the limits 0.6 $0.8 \mathrm{eV}[1,12,27]$. But in the work [23], it was found that this level is by $0.47 \mathrm{eV}$ higher than the top of the valence band. As the accuracy of determination of positions inherent to energy levels by quantum-mechanic methods is not high, in our calculations we used respective values of ionization energies observed experimentally. For the vacancy of cation, we used the values 0.05 and $0.8 \mathrm{eV}$ according to the first and second charge states. There is a little information about the position of ionization levels for the inersticial tellurium atom. We used the value $0.15 \mathrm{eV}$ that was also used by Chern [2] for the first level and $0.57 \mathrm{eV}$ for the second level of ionization. The value $0.57 \mathrm{eV}$ was obtained in calculations [16]. The level with the ionization energy close to this value $(0.583 \mathrm{eV})$ was fixed in the work [24], and in opinion of the authors belongs to the intersticial atom of tellurium.

There are no large divergences between the values of ionization energies for the intersticial cadmium atom that were offered by different authors. The levels of $E_{c}$ $(\approx 0.02) \mathrm{eV}$ and $E_{c}-(\approx 0.2) \mathrm{eV}$ that are observed in the material $[17,18]$ were attributed by the authors according to the first and second charge states of intersticial cadmium atom. The calculation [12] gives approximately the same results, which confirms that these values can be considered as the first and second ionization levels of $\mathrm{Cd}_{i}$. For the vacancy of tellurium, there are a few variants of energy levels location in the forbidden gap $[2,12,16,25,26]$. We considered that the ionization energies of the tellurium vacancy are $E_{c}$ $0.01 \mathrm{eV}$ and $E_{c}-0.34 \mathrm{eV}$ according to the first and the second levels.

\section{Results and discussions}

Using the deduced formulas, we performed calculation of dependences for concentrations of point defects and free charge carriers in CdTe on the temperature $T$ and partial pressure of cadmium vapors $P_{\mathrm{Cd}}$ under twotemperature annealing. The results have been presented in Figs 1 to 4. As it is obvious from Figs 1 and 2, the theoretically determined concentration of electrons $n$ describes experimental data satisfactorily, which testifies to adequacy of the accepted model.

According to the calculation, at low pressures of cadmium vapors $P_{\mathrm{Cd}}$ at two-temperature annealing, material should possess $p$-type conductivity. When annealing, the increase in $P_{\mathrm{Cd}}$ results in diminishing the hole concentration, thermodynamic $p$ - $n$ transition and obtaining the material with the $n$-type conductivity (Fig. 2). 


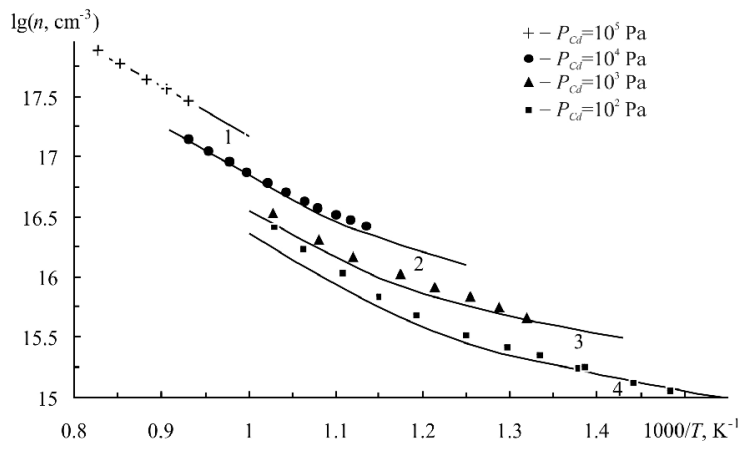

Fig. 1. Dependence of the electron concentration on the annealing temperature $T$ at the partial pressures of cadmium vapor $P_{\mathrm{Cd}}$. Lines - calculation, points - experiment: $1-10^{5}$, $2-10^{4}, 3-10^{3}, 4-10^{2} \mathrm{~Pa}$.

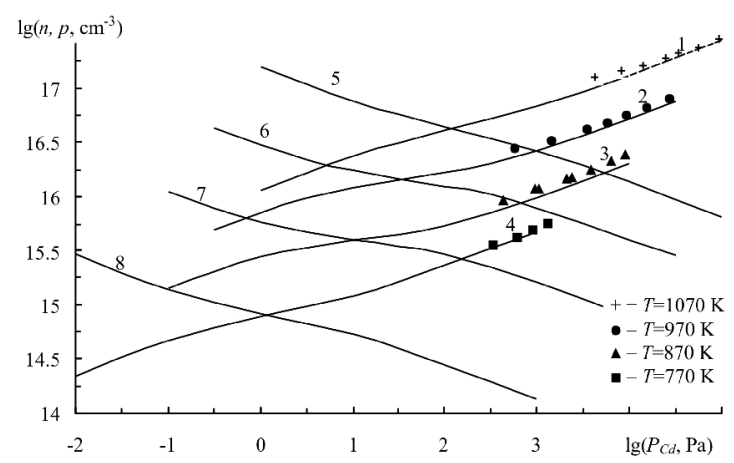

Fig. 2. Dependence of electron $n$ and hole $p$ concentrations on the partial pressure of cadmium vapor $P_{\mathrm{Cd}}$. Lines - calculation, points - experiment. For $n: 1-1070,2-970,3-870,4-$ $1070 \mathrm{~K}$; for $p: 5-1070,6-970,7-870,8-770 \mathrm{~K})$.

There are two temperature intervals in material of $n$-type conductivity, within the limits of which different defects prevail (Fig. 3). Up to the temperature $T \approx 900 \mathrm{~K}$, the dominant donor is the doubly ionized vacancy of tellurium $V_{\mathrm{Te}}^{2+}$ (Fig. 3a). At the temperatures $T>900 \mathrm{~K}$, the doubly ionized intersticial atom of cadmium $\mathrm{Cd}_{i}^{2+}$ prevails (Fig. 3b). These results are in accord with other works $[2,6]$.

At the temperatures $T<1000 \mathrm{~K}$, the dominant acceptor in material of $p$-type is the singly charged cadmium vacancy $V_{\mathrm{Cd}}^{-}$(Fig. 3a). With the growth of temperature, the concentration of intersticial tellurium atoms grows quicker than the concentration of cadmium vacancies, and at the temperatures $T>1000 \mathrm{~K}$ intersticial tellurium atom $\mathrm{Te}_{i}^{-}$determines the $p$-type of material conductivity (Fig. 3b).

The complicated defect structure in the crystals CdTe is observed at maximal pressure of cadmium vapors. At the temperatures of $T<900 \mathrm{~K}$, the dominant donor defect is the vacancy of tellurium $V_{\mathrm{Te}}^{2+}$, and at higher temperatures it is the intersticial cadmium atom $\mathrm{Cd}_{i}^{2+}$. At the temperatures $T>1100 \mathrm{~K}$, the concentrations of singly ionized intersticial cadmium atoms $\mathrm{Cd}_{i}^{+}$are comparable with the concentrations of $\mathrm{Cd}_{i}^{2+}$. Therefore, to interpret experimental dependences at $T>1100 \mathrm{~K}$ it is necessary to take into account the existence of intersticial cadmium atom in two charge states: $\mathrm{Cd}_{i}^{+}$and $\mathrm{Cd}_{i}^{2+}$.

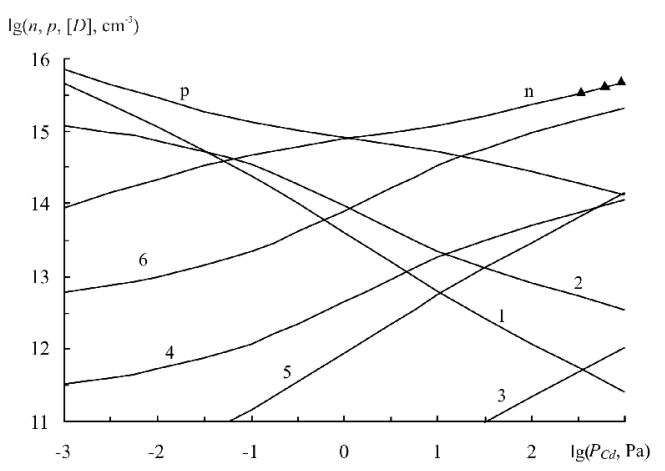

a)

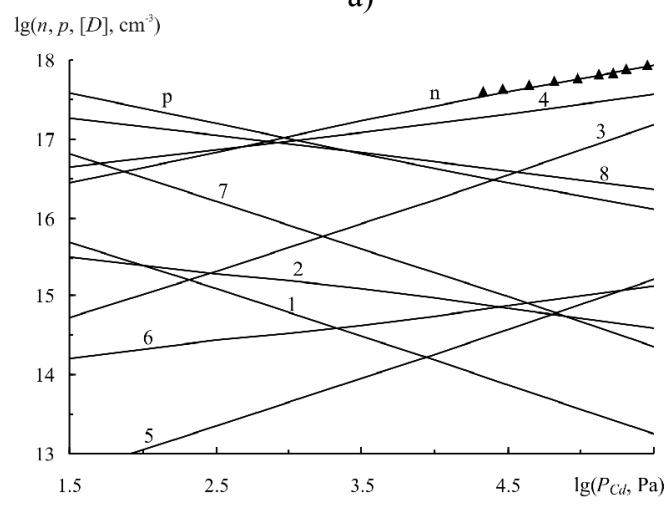

b)

Fig. 3. Dependence of point defects [D], electron $n$ and hole $p$ concentrations on the partial pressure of cadmium vapor $P_{\mathrm{Cd}}$. Lines - calculation, points - experiment. $1-V_{\mathrm{Cd}}^{-}, 2-V_{\mathrm{Cd}}^{2-}$, $3-\mathrm{Cd}_{i}^{+}, 4-\mathrm{Cd}_{i}^{2+}, 5-V_{\mathrm{Te}}^{+}, 6-V_{\mathrm{Te}}^{2+}, 7-\mathrm{Te}_{i}^{-}, 8-\mathrm{Te}_{i}^{2-}$. (a $-T=770 \mathrm{~K} ; \mathrm{b}-T=1170 \mathrm{~K}$.)

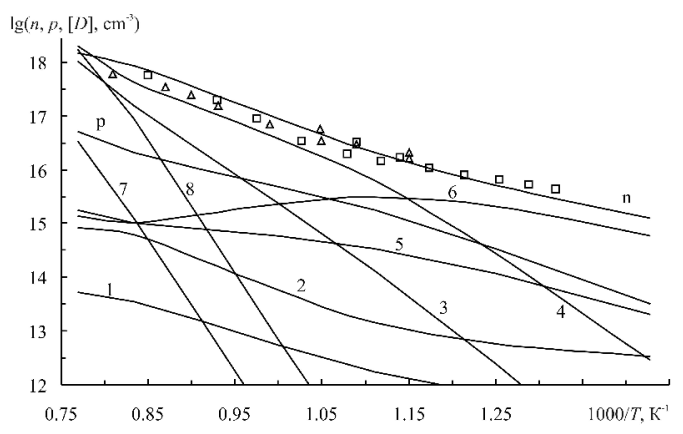

Fig. 4. Dependence of point defects $[D]$, electron $n$ and hole $p$ concentrations on the annealing temperature at the maximal pressure of cadmium vapor $P_{\mathrm{Cd}}$. Line - calculation, points experiment. $1-V_{\mathrm{Cd}}^{-}, 2-V_{\mathrm{Cd}}^{2-}, 3-\mathrm{Cd}_{i}^{+}, 4-\mathrm{Cd}_{i}^{2+}, 5-V_{\mathrm{Te}}^{+}$, $6-V_{\mathrm{Te}}^{2+}, 7-\mathrm{Te}_{i}^{-}, 8-\mathrm{Te}_{i}^{2-}$. 


\section{Conclusion}

Using the method of thermodynamic potentials, we described the processes of defects creation in the crystals of CdTe depending on the technological parameters (temperatures $T$ and partial pressure of cadmium vapors $P_{\mathrm{Cd}}$ ) of the two-temperature annealing. The concentrations of atomic point defects and free charge carriers were calculated as dependent on $T$ and $P_{\mathrm{Cd}}$.

Also determined are the ranges of prevailing donor and acceptor defects that determine the type of material conductivity.

For all the explored values of annealing temperatures $670-1200 \mathrm{~K}$ and partial pressures of cadmium vapors $P_{\mathrm{Cd}} 10^{1}-10^{5} \mathrm{~Pa}$, the dominant defects are charged.

The results obtained in the work can be used by specialists in the field of device designing and physicistsexperimenters with the purpose to establish optimum parameters of after-growth annealing for preparation of crystals with the electrophysical properties set in advance.

\section{References}

1. D. de Nobel, Phase equilibrium and semiconductor properties CdTe // Phil. Res. Repts. 14, p. 361-399 (1959).

2. S.S. Chern, H.R. Vydyanath, F.A. Kroger, The defect structure of CdTe: Hall data // J. Solid State Chem. 14, p. 33-43 (1975).

3. V.M. Glazov, L.M. Pavlova, Homogeneity region in the system based on cadmium telluride // Neorganicheskiye materialy 30(5), p. 629-634 (1994), in Russian.

4. P. Fochuk, O. Korovyanko, O. Panchuk, High-temperature point defect equilibrium in CdTe modelling // J. Cryst. Growth 197, p. 603-606 (1999).

5. P.M. Fochuk, O.O. Korovyanko, O.E. Panchuk, Calculation of incorporation constants for doping elements in CdTe // Physics and chemistry of solid state 2(3), p. 475-480 (2001), in Ukrainian.

6. P.M. Fochuk, O.E. Panchuk, L.P. Scherbak, Dominating points defects in CdTe crystals: the range of Cd saturation // Physics and chemistry of solid state 5(1), p. 136-141 (2004), in Ukrainian.

7. P. Fochuk, R. Grill, O. Panchuk, The nature of point defects in CdTe // Journal of Electronic Materials. 35(6), p. 1354-1359 (2006).

8. D.M. Freik, V.V. Prokopiv, U.M. Pysklynets, Atomic defects and their compensation in the doped and undoped cadmium telluride // Physics and chemistry of solid state 4(3), p. 547-555 (2004), in Ukrainian.

9. R. Grill, A. Zappettini, Point defects and diffusion in cadmium telluride // Progress in Crystal Growth and Characterization of Materials 48/49, p. 209-244 (2004).

10. R. Grill, J. Franc, P. Höschl, I. Turkevych, E. Belas, and P. Moravec, Semi-insulating Tesaturated CdTe // IEEE Trans. Nucl. Sci. 52(5), p. 1925-1931 (2005).
11. Li Yujie, Ma Guoli, Jie Wanqi, Point defects in CdTe // J. Cryst. Growth 256 p. 266-275 (2003).

12. M.A. Berding, Native defects in CdTe // Phys. Rev. 60(12), p. 8943-8950 (1999).

13. V.G. Levich, Course of Theoretical Physics, vol. V1. Nauka, Moscow, 1969 (in Russian).

14. Yu.B. Rumer, M.Sh. Ryvkin, Thermodynamics, Statistical Physics and Kinetics. Nauka, Moscow, 1972 (in Russian).

15. G. Korn, T. Korn, Reference Book on Mathematics. Nauka, Moscow, 1977, (in Russian).

16. Su-Huai Wei and S.B. Zhang, Chemical trends of defect formation and doping limit in II-VI semiconductors: The case of CdTe // Phys. Rev. B 66, 155211-1-155211-10 (2002).

17. A.V. Savitskiy, B.I. Tkachuk, P.N. Tkachuk, Electric properties of extracted cadmium telluride // Fizika tekhnika poluprovodnikov 26(5), p. 952-955 (1992), in Russian.

18. E.A. Bobrova, Y.V. Klevkov, S.A. Medvedev, A.F. Plotnikov, Investigation of the deep electron states in textured polycrystals $p$-CdTe of stoichiometric composition by the DLTS method // Fizika tekhnika poluprovodnikov 36(12), p. 14261431 (2002), in Russian.

19. V. Babentsov, V. Corregidor, K. Benz, M. Fiederle, T. Feltgen, E. Dieguez, Defect engineering in CdTe based on the total energies of elementary defects // Nucl. Instr. Meth. Phys. Res. A 458, p. 85-89 (2001).

20. V.S. Ivanov, V.B. Stopachynskyj, V.A. Chapnin, Differential spectroscopy of local centers in CdTe // Fizika tekhnika poluprovodnikov 5(1), p. 101-105 (1971), in Russian.

21. N.V. Agrinskaya, O.A. Matveev, A.V. Nikitin, V.A. Sladkova, Features of edge photoluminescence of CdTe-Cl crystals // Fizika tekhnika poluprovodnikov 21(4), p. 676-679 (1987), in Russian.

22. A.V. Savitskiy, O.A. Parfenyuk, M.I. Ilaschuk, A.J. Savchuk, S.N. Chupyra, Characteristic equilibrium and low-temperature photoluminescence of monocrystalline CdTe-Pb // Fizika tekhnika poluprovodnikov 38(5), p. 516-521 (2004), in Russian.

23. B.K. Meyer, W. Stadler, Native defect identification in II-VI materials // J. Cryst. Growth 161, p. 119-127 (1996).

24. K. Scholz, H. Stiens, G. Muller-Vogt, Investigations on the effect of contacts on p-type CdTe DLTSmeasurements // J. Cryst. Growth 197, p. 586-586 (1999).

25. W. Stadler, D.M. Hofmann, H.C. Alt, T. Muschik, B.K. Meyer, Optical investigation of defects in CdZnTe // Phys. Rev. B 51(16), p. 10619-10630 (1995).

26. S. Lany, V. Ostheimer, H. Wolf, Th. Wichert, Vacancies in CdTe: experiment and theory // Physica B 308-310, p. 958-962 (2001).

27. F.T.J. Smith, Electrically active point defects in cadmium telluride // Metallurgical and Material Trans. B 1(3), p. 617-621 (1970). 